\title{
A methodology to facilitate knowledge sharing in the new product development process
}

\author{
D. J. BRADFIELD $\dagger$ and J. X. GAO* \\ $\dagger$ Centre for Decision Engineering, Department of Manufacturing, Cranfield University, \\ Bedfordshire, MK43 0AL, UK \\ $\$$ School of Engineering, University of Greenwich at Medway, Chatham Maritime, \\ Kent, ME4 4TB, UK
}

\begin{abstract}
This paper describes the findings of an exploratory case study to investigate knowledge sharing problems in the new product development (NPD) process of a multi-national manufacturing company, and classifies the problems into three categories, i.e. (a) the lack of an explicit definition and prioritization of information about the knowledge used in the NPD process, (b) the challenges raised by, and lack of tools to support, knowledge sharing in a multilingual, multidisciplinary environment, and (c) the dissemination of information about the task and process knowledge to process users. An ontology-based methodology has been proposed, and a pilot study has been carried out to solve these problems. The pilot study includes the selection of suitable candidate tasks (or sub-processes) for the study, the elicitation of information about the selected task knowledge, the development of task knowledge ontology, and a mechanism to visualize and disseminate the ontology to process users. Early implementation and tests have shown that the proposed methodology may be used to facilitate knowledge sharing in the new product development process. The project was sponsored by a leading heating system manufacturer and further tests will be carried out with real industrial problems.
\end{abstract}

Keywords: Knowledge sharing; New product development; Multilingual; Ontology; Business process

\section{Introduction}

As new products become the focus of competition for many manufacturers, the new product development process becomes increasingly important to these businesses (Brown and Eisenhardt 1995, Ramesh and Tiwana 1999). Furthermore, an increasing need by manufacturing companies to compete on quality and time to market has made the effective sharing and transfer of product development process knowledge into a means of achieving a competitive advantage (Ramesh and Tiwana 1999, Gieskes and Langenberg 2001). Consequently, great attention has been focused in recent years on the application of knowledge management to new product

*Corresponding author. Email: j.gao@gre.ac.uk 
development (Zahay et al. 2004). The product development process is comprised of, 'a sequence of steps or activities which an enterprise employs to conceive, design, and commercialise a product'(Ulrich and Eppinger 2003). These activities are linked by an exchange of information (Browning and Eppinger 2002). Indeed, Eppinger states that this exchange of information, '.. is the lifeblood of product development' (Eppinger 2001).

Product development demands the cooperation of people from different parts of the organization with different expertise and varying levels of experience. As a result effective communication is required to manage the activities in the New Product Development (NPD) process (Effendi et al. 2002). The emergence of the multinational corporation with a network of geographically dispersed operations, as described by Ghoshal and Bartlett, has complicated this issue still further (Ghoshal and Bartlett 1990). Product development project teams often consist of members located in different countries and they face various communication challenges, among them differences in language and culture (Morelli et al. 1995, Desouza and Evaristo 2003). These challenges prompted an investigation of knowledge sharing problems at a multinational manufacturing company.

\section{Investigation of industrial requirements}

\subsection{Introduction}

In 2003, a three-year project was embarked upon with a medium-sized, marketleading manufacturer of electromechanical goods. The aim of the collaboration was to develop and implement a long-term knowledge management strategy. Notably, the company is the product of a recent merger between German and Anglo-French companies competing in the same sector. New products developed by the company are of two types: changes to existing product lines in order to accommodate environmental legislation and upgrades and changes to existing products. The manufacturing and research and development (R\&D) operations in the company are located on main sites in three European countries, each site speaking a different language.

New products play a key role in the company's business. More than $4 \%$ of its employees work in research and development roles. Nonetheless, the company's research activity is mainly of an applied nature. As part of an effort to improve the quality of its product development process, the company created a business process map that describes all the new product development activities. These activities span the entire product development process, ranging from product strategy to production. It was intended that the process should capture best practice from formal NPD processes previously used within the company, as well as analogous processes employed by competitors. The New Product Development Business Process (NPDBP) consists of seven phases and around a hundred sub processes. Each sub process is broken down into a number of tasks. Documentation of the process was available in paper format and in digital format on the company's Intranet. Three process owners are appointed for each sub process. Most of the processes are intended for use on more than one site and in these instances, a process owner for each site is appointed. 
An exploratory study was carried out in order to gain a basic understanding of the knowledge sharing challenges surrounding the NPDBP and provide a foundation for identifying the research problems. The aims of the exploratory research were:

1. To obtain information about how knowledge (information) flows in the NPDBP.

2. To establish whether the employees using the NPDBP (hereafter referred to as 'users') believed that they were experiencing knowledge sharing problems.

3. To establish what kind (if any) of knowledge-sharing problems the users were experiencing.

\subsection{Method of investigation}

Three research methods were employed to achieve these aims. The first method involved reference to the NPDBP documentation and other company documentation, including internal presentation slides, thus addressing the first aim. The second method was to carry out unstructured interviews with a selection of sub process owners. The third method was to perform a knowledge audit by means of structured interviews with the same selection of NPDBP sub process owners used for the unstructured interviews, thereby addressing all three aims.

In the case of the first method, the complete documentation for the NPDBP was secured, including business process flow maps for every detailed process. Additionally, the results of an internal company-wide NPDBP user feedback survey were obtained. The company's internal survey exercise involved interviews with detail process owners from the three sites and the documentation included extensive quotations from NPDBP users. The quotations and comments were examined and any knowledge problems were identified and broadly categorized. In the case of the second method exploratory interviews were carried out with eight sub process owners representing all seven phases of the NPDBP. Interviews were conducted in an 'unstructured' fashion. Analysis of the interview data involved identifying the main knowledge sharing problems mentioned by the interviewees and grouping them into broader categories. Finally, execution of the third method involved structured interviews with the same eight process owners used for the exploratory interviews already discussed. The questions used in the interviews were adapted from those employed in a knowledge audit study described by Liebowitz et al. (2000).

\subsection{Findings}

Although the company intends that the NPDBP will be adopted on a group-wide basis, the majority of its initial development was undertaken at one site. Consequently, many of the processes contained in the NPDBP documentation are modelled on practices and cultural approaches used at the German site, which reportedly differed in some aspects from those encountered at the British and French sites. Furthermore, the majority of the process documentation was originally written in German and then translated into English for distribution to the British and French operations. The key findings from the review of company literature and exploratory interviews were divided into broad problem categories, which are listed 
in table 1. It is worth noting that there were a number of other problem types, but these will not be the focus of this study.

As seen in table 1, category A refers to the lack of an explicit definition and prioritization of information about the knowledge used in the NPD process. One example taken from the interviews of information that has not been explicitly defined is information about the required input knowledge and output knowledge for a given task. These knowledge inputs or outputs could be of an explicit nature, such as a test report, or of a tacit nature, such as advice from an expert in the domain of interest. This is because many of the decisions taken in the course of a product development activity are dependent on the information generated by other activities, as claimed by Browning and Eppinger (2002). A further example was the difficulty of discovering information about who the experts for a given task were. In this case, an expert is an individual with what was considered extensive experience of a technical area, or those who had been a member of a project where similar problems had been encountered in the past. Where some indication of the knowledge required to complete a task is available, there is no indication of the importance of that knowledge in the context of that task. In short, this lack of an explicit definition of the knowledge required executing a sub process or task appears to hinder the development of a shared understanding among users of the NPD process.

Category B refers to the challenges raised by a multilingual, multidisciplinary environment. Two types of challenge were determined. Challenge type one concerns the challenges presented by different vocabularies used by the various engineers, designers and different functional disciplines involved in the NPD. This is reflected by work already carried out in the NPD domain by Holland et al. (2000) and in the requirements engineering domain by Effendi et al. (2002) and Kerr et al. (2004). Kerr et al. (2004) posit that functional disciplines involved in the design process stages, 'bring their own language, jargon and perspectives to a design project'. A simple example of this from the exploratory study is the German phrase for the part

Table 1. Key findings from review of company literature and exploratory interviews.

Key findings

Problem category description Problem category

Roles and responsibilities connected to process tasks are missing or unclear.

No explicit definition and A

Experts for tasks and processes are not defined. prioritization of the process knowledge.

Knowledge required as inputs for a process are missing/difficult to find.

Unclear what the 'minimum knowledge' required is.

Inter-lingual translation of some process descriptions is poor.

Existing NPD process knowledge on Intranet difficult to find or unavailable.

Intranet IP representation unintuitive, non-interactive and difficult to navigate.

Lack of tools to support multilingual knowledge environment.

Difficulties in communicating the knowledge
B

C 
'nozzle holder', which is known as 'Kammerträger' by some engineers and 'Düsenstock' by others. Challenge type two concerns the more obvious problem of linguistics; that is, instances where the information or knowledge is available in a language that cannot be understood by those persons that require it. This issue affects both explicit knowledge sources, such as documents and databases, and sources of tacit knowledge like experts, who may not be able to communicate in the required language. The overall message is that language differences of either type make it difficult for those people involved in the new product development process, essentially the process users, to achieve what Kleinsmann and Valkenburg (2005) refer to as 'a shared viewpoint'.

Category $\mathrm{C}$ concerns the dissemination of information about the task and process knowledge to process users. Here, dissemination means the distribution and sharing of information among groups via various mediums, as defined by Akgün et al. (2006). Examples of information about the knowledge that might be sought include the subsequent use of knowledge generated by a task, and information about the experts for a task or sub process. There is a lack of a mechanism to disseminate or 'push' information about the knowledge to the process users. This means that significant quantities of time are spent searching for and collating information, which ranged from a few hours to a working week in each NPD project.

\section{Academic research addressing the industrial problems}

By use of the exploratory study in industry, a need has been identified to facilitate the sharing of knowledge in the new product development process of the multinational manufacturing organization. A knowledge-sharing technology approach has been taken to address all three problem categories.

Problem A is essentially about achieving a shared understanding among people of information about the knowledge associated with tasks and sub processes in the new product development process. These problems are being tackled through the development of a knowledge-based tool built around ontology. Studer et al. (1998) define ontology as a formal, explicit, specification of a shared conceptualization. A conceptualization is an abstract, simplified view of the world that we wish to represent for some purpose (Gruber 1993). Ontologies may be employed to facilitate a shared understanding of a knowledge domain that may be communicated among people (Pinto and Martins 2004). Reference to the literature reveals that ontology-based approaches have already been applied to other problems in the new product development process, such as NPD process management in Moore et al. (1999) and information sharing protocols for translating customer needs into product specifications in Rezayat (2000). The SHARE project proposed using ontology in the classification and organization of various forms of design information that could be captured electronically (Toye et al. 1994). Ramesh and Tiwana (1999) describe a prototype system to capture and manage NPD process knowledge. None of these approaches addresses all the knowledge-sharing problem categories presented here, as shown in table 2 .

In order to address problem $\mathrm{B}$, a mechanism is needed to make information about the knowledge accessible to process users in a multidisciplinary, multilingual environment. As with problem A, an ontology-based solution is proffered, in this 


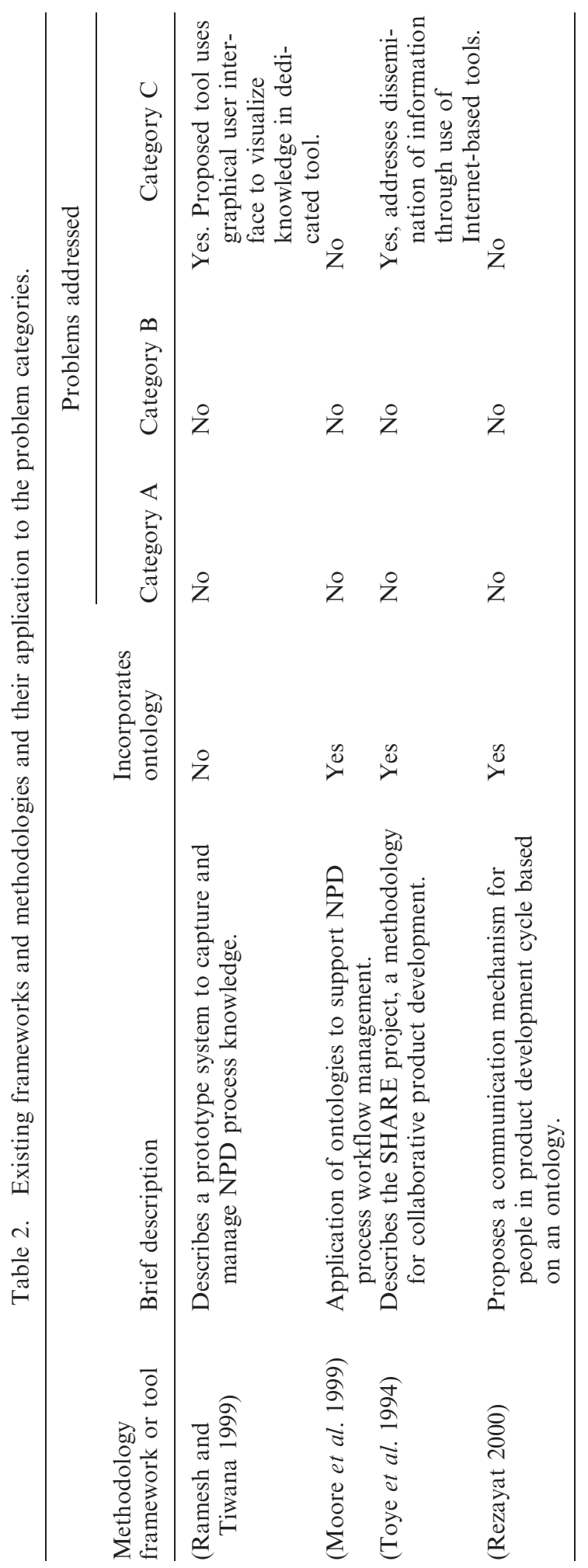


case using a multilingual ontology. Guyot et al. (2005) define a multilingual ontology as one that includes a set of dictionaries for each of the languages required by its users. It functions by mapping keywords from these languages to the same concept in the ontology. A portal may then be constructed to retrieve the same information from the ontology, regardless of the language (Lauser et al. 2002). This approach is applicable to both the issue of sharing the ontology among users speaking different languages and the issue of semantic differences between vocabularies used by different functions or those with different educations and view of design, termed 'design identities' by Kilker (1999). While surveys in the literature (Pinto and Martins 2004, Corcho et al. 2003) identify a range of generic methodologies for developing ontologies, methodologies for the development of multilingual ontologies are far fewer in number. A framework for building a multilingual ontology is presented by Lauser et al. (2002) and a method for developing a bilingual ontology is given by Carpuat et al. (2002). Multilingual ontologies may be represented in languages such as RDFS (Lauser et al. 2002) or related languages.

Problem $\mathrm{C}$ has been addressed by providing a mechanism to disseminate information within a multidisciplinary multilingual environment. An Internet Web-based solution has been used to achieve this. Internet-based visualization techniques have already been identified by Zhang et al. (2004) as a basis for the sharing of information sharing in collaborative product design and manufacturing. Zhang et al. (2004) also argue that web-based tools 'are desirable to adapt geographically multidisciplinary product development teams...'.

\section{A knowledge sharing methodology and pilot case study}

\subsection{Introduction}

A pilot study was carried out with the aim of developing a knowledge-based tool for the case of the knowledge associated with a single sub process and task in the case study company's new product development process. The methods employed are described, followed by a discussion of the salient features of the resulting tool. Together, the ontology and mechanisms form the basis of a knowledge-based tool. Two parts of methodology have not been applied to this case. These parts are the mechanism for the presentation of the ontology in different languages and the mechanism for the prioritization of task level knowledge.

The methodology used in the pilot study consisted of four stages (see figure 1):

1. Selection of a suitable candidate sub process and task for the study.

2. Elicitation of information about the task and sub process knowledge.

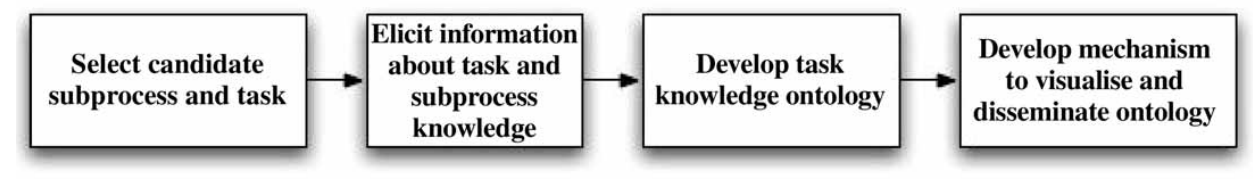

Figure 1. Overview of methodology used to develop knowledge-based tool. 
3. Development of ontology of task knowledge.

4. Development of a mechanism to visualize and disseminate the ontology to process users.

\subsection{Selection of a candidate process and task}

Selection of the candidate detail process and detail process task was made according to four criteria: the first criterion was that the process and task should involve the use of knowledge from a range of functions (Ulrich and Eppinger 2003), such as marketing, design and manufacturing, involved in the new product development process. Although it was unlikely that knowledge from all functions contributing to the NDP process could be represented, it was considered that the inclusion of more than one would provide a broader indication of the spectrum of knowledge present in the NPD knowledge domain. The second criterion was that the candidate task has a knowledge output that is used by other tasks in the NPD process. On a more practical note, the third criterion is that the candidate process should be one of those studied in the knowledge audit, in order to exploit the results of that study. Finally, the fourth criterion is that the designated expert for the task was both available and willing to take part in the case study exercise.

\subsection{Identification of the task knowledge}

Information about the task knowledge required to develop the ontology was elicited from three sources. The first source was the knowledge audit study of eight sub-processes in the company NPDBP using a simplified version of the knowledge audit steps proposed by Liebowitz et al. (2000). This study involved semi-structured interviews with one of the three owners for each process. Each of these owners had been nominated by the company as a process expert. The second source was a semistructured interview with the owner of the selected process to understand the knowledge associated with a single task in that process. Finally, the third source was the company's NPDBP documentation, which included a business process map depicting the selected process and task, as well as descriptions of some of the data and information needed for some processes.

\subsection{Development of a task knowledge ontology}

The ontology of task knowledge was developed using the steps outlined by Noy and McGuinness (2001), These steps are:

- Determine the domain and scope of the ontology.

- Consider reusing existing ontologies.

- Enumerate important terms in the ontology.

- Define the classes and the class hierarchy.

- Define the properties of classes-slots.

- Define the facets of the slots.

- Create instances.

The methods used to elicit the information required to develop the ontology are discussed above. Implementation of the ontology was achieved using the 
frame-based implementation of the Protégé ontology editor developed at the Stanford University School of Medicine (Stanford Medical Informatics 2006a). The version used was Protégé 3.0. Evaluation studies by Duineveld et al. (2000) and more recently by Lambrix et al. (2003) have concluded that this tool is among the easiest to learn and use of the available ontology editing tools, which was an important factor given the scope of this study. In addition, it provides the functionality to build the simple knowledge-based tool envisaged for this study and supports a range of ontology representation languages and plug-in functionalities which may be required in future research (Corcho et al. 2003). Protégé uses a frame-based knowledge model in which ontologies are comprised of classes, which represent concepts in the domain, slots, which describe the properties and attributes of classes, facets, which describe properties of slots, and finally axioms, which specify additional constraints (Noy et al. 2000).

\subsection{Development of a mechanism to enable the visualization and dissemination of the ontology}

Development of a mechanism to visualize and disseminate the task knowledge ontology was realized in two stages:

1. Development of the ontology visualization mechanism.

2. Development of a dissemination mechanism.

Combined with the ontology, the mechanisms formed the basis of a simple knowledge-based tool to share information about the knowledge associated with a task from the new product development process.

Stage one involved developing a simple knowledge-based tool using the Protégé editor in order to capture the knowledge for the 'Analysis of competitor products from customer viewpoint' task. This involved creating instances of the classes defined in the ontology using the information elicited from the interviews and business process documentation. Following this, forms were created for each class to represent the information for each of the slots created for that class. In this way, a user interface is constructed that allow a user to view and navigate the information contained in the ontology.

The Protégé ontology software tool used to develop the ontology includes a number of built-in features and plug-ins that allow the ontology to be visualized and browsed. However, the use of these features or plug-ins demands that the Protégé tool is installed locally on a user's computer. This situation may restrict access to the tool in a business environment. One software tool that is already accessible to all the NPDBP users in the case study company is the Microsoft Internet Explorer ${ }^{\circledR}$ web-browser client. Stage two then involved constructing a browser-based tool using the WebProtégé software included with the Protégé ontology editor (Stanford Medical Informatics 2006b). This tool provides a means of browsing and editing ontology through a web-browser client. Web Protégé is a Java-based application that is deployed from a server application. The tool was developed according to the guidelines in the tutorial provided with WebProtégé software. 


\subsection{Results of the pilot study}

Having reviewed the sub-process analysed in the knowledge audit, it was decided to select a sub-process called 'Definition of marketing requirements' and a task belonging to this process entitled 'Comparison of competitor products from customer viewpoint'. The process is part of the conception phase of the NPDBP and both the process and its constituent tasks demand the sharing of knowledge between different functions of the company, as suggested by Ulrich and Eppinger (2003) and Zahay et al. (2004). Additionally, the selected task requires both knowledge 'inputs' and 'outputs' and a process owner who was willing and able to be interviewed. In this way, all four of the selection criteria were satisfied.

It was intended that the ontology would provide the user performing the selected process task with information about the knowledge associated with that task. Eppler et al. (1999) proposed that there are three kinds of business process knowledge: knowledge about the process, knowledge within the process and knowledge from the process. Knowledge about the process may be in both explicit form, such as flow maps showing the process stages, and implicit forms like the experience possessed by the process owners of managing the activities in that process. Some of this type of knowledge is encoded in the business process documentation of the company. Knowledge within the process is that which is generated during the execution of the process. This knowledge will typically be in an explicit form and includes documents such as test reports, project summary reports and decisions recorded in meeting minutes or in an email. The knowledge derived from a process is the experience that has been acquired from having carried out that process, e.g. lessons learned, the experience accumulated by different people and improvements that could be implemented in future projects. The concentration in this pilot study will be on knowledge within the process.

Figure 2 shows the taxonomy used as the basis for constructing the ontology. Given that the focus of this study is the methodology, only three of the classes in the figure will be discussed here, namely, 'NPD process task', 'Knowledge item' and 'Actor'.

Consider first the 'NPD process task' class. The questions that might be posed by a task user about the task might include:

- What knowledge is required to execute this task?

- What knowledge is generated by this task?

- Who are the experts for this task?

These questions may be viewed as the competency questions (Noy and McGuinness 2001) that the ontology must answer and they were used to determine the relationships or slots created for the 'NPD process task' class, as shown in figure 3. For example, the requires_knowledge_slot' provides a relationship to link an instance of the 'NPD process task' class to an instance of the 'Knowledge item class'.

Instances of the 'Knowledge item' class are chunks of knowledge (Debenham and Clark 1994), which may include a technical report, guidelines for using a software tool or advice from an expert on an issue associated with the task. Some of the competency questions a user could pose about a knowledge item include:

- What task generates this knowledge item?

- For what tasks does this knowledge item provide knowledge? 


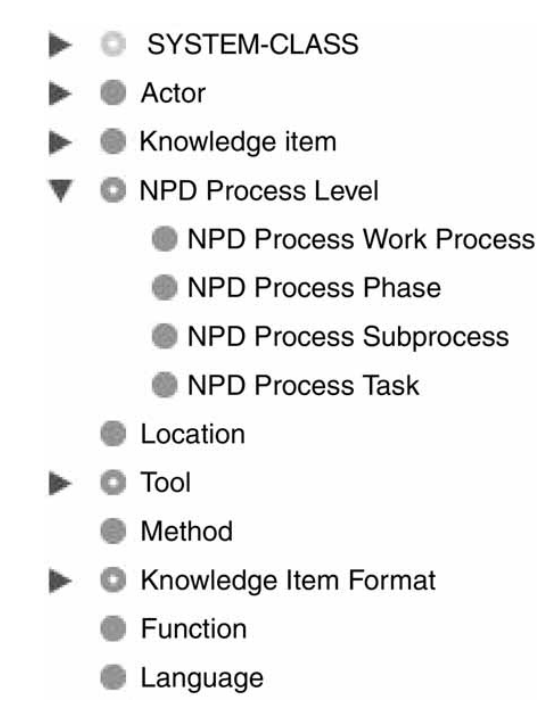

Figure 2. Classes in the NPD process knowledge ontology.

\begin{tabular}{|l|l|l|}
\hline \multicolumn{1}{|c}{ Name } & Cardinality & \multicolumn{1}{c|}{ Type } \\
\hline generates_knowledge_item & multiple & Instance of Knowledge Item \\
has_expert & multiple & Instance of Actor \\
has_knowledge_contributor & multiple & Instance of Actor \\
involves_function & multiple & Instance of Function \\
is_part_of_subprocess & single & Instance of NPD Process Subprocess \\
requires_knowledge_item & multiple & Instance of Knowledge Item \\
task_aim_purpose & single & String \\
task_description & single & String \\
task_number & single & String \\
task_title & single & String \\
\end{tabular}

Figure 3. Slots or relationships for the 'NPD process task' class.

- Where is the knowledge item located?

- In what languages is the knowledge available?

As in the previous case, the competency questions served as the basis for formulating the slots for the 'Knowledge item' class, illustrated in figure 4. For example, the 'generated_by_task' slot provides a relationship to link an instance of the 'Knowledge item' class to relevant instances of the 'NPD process task' class. Lastly, consider the 'Actor' class. In this context, actors may be defined as human sources of knowledge or 'knowledge brokers'. Instances of the 'Actors' include process owners, quality method experts and various task knowledge contributors. 


\begin{tabular}{|lllll|}
\hline \multicolumn{1}{|c}{ Name } & Cardinality & \multicolumn{1}{c|}{ Type } \\
\hline available_in_language & multiple & \multicolumn{1}{c|}{ Instance of Language } \\
generated_by_task & multiple & \multicolumn{1}{c}{ Instance of NPD Process Task } \\
has_owner & single & Instance of Actor \\
knowledge_item_description & multiple & String \\
knowledge_item_format & multiple & Instance of Knowledge Item Format \\
knowledge_item_location & multiple & Instance of Location \\
knowledge_item_title & single & String \\
provides_knowledge_for_task & multiple & Instance of NPD Process Task
\end{tabular}

Figure 4. Slots or relationships for the 'knowledge item' class.

\begin{tabular}{|l|lll|}
\hline \multicolumn{1}{|c}{ Name } & Cardinality & \multicolumn{1}{c|}{ Type } \\
\hline actor_name & single & String \\
contributes_knowledge_to_task & multiple & Instance of NPD Process Task \\
expert_for_method & multiple & Instance of Method \\
expert_for_task & multiple & Instance of NPD Process Task \\
has_location & multiple & Instance of Location \\
owner_for_knowledge_item & multiple & Instance of Knowledge Item \\
owner_for_subprocess & multiple & Instance of NPD Proccess Subprocess
\end{tabular}

Figure 5. Slots or relationships for the 'actor' class.

The knowledge they provide is likely to be in a tacit form like experience or expertise. Some competency questions pertinent to the 'Actor' class include:

- To what tasks does the actor contribute knowledge?

- For what tasks is the actor declared an expert?

- What sub-processes does the actor own?

- What is the geographical location of the expert?

Once again, the competency questions served as the basis for formulating the slots for the 'Actor' class (see figure 5). For example, the 'contributes_to_task' slot provides a relationship to link an instance of the 'Actor' class to relevant instances of the 'NPD process task' class.

\subsection{Dissemination and visualization mechanisms}

Results from the implementation of method to provide a mechanism for visualizing and disseminating the task knowledge are documented in a series of screenshots. Figure 6 illustrates the browser window and forms that constitute the knowledgesharing tool built using the Protégé editor. This acts as the basis of the visualization mechanism. Figure 7 shows the task knowledge window, which provides information about the knowledge required for and generated by the case study task. 


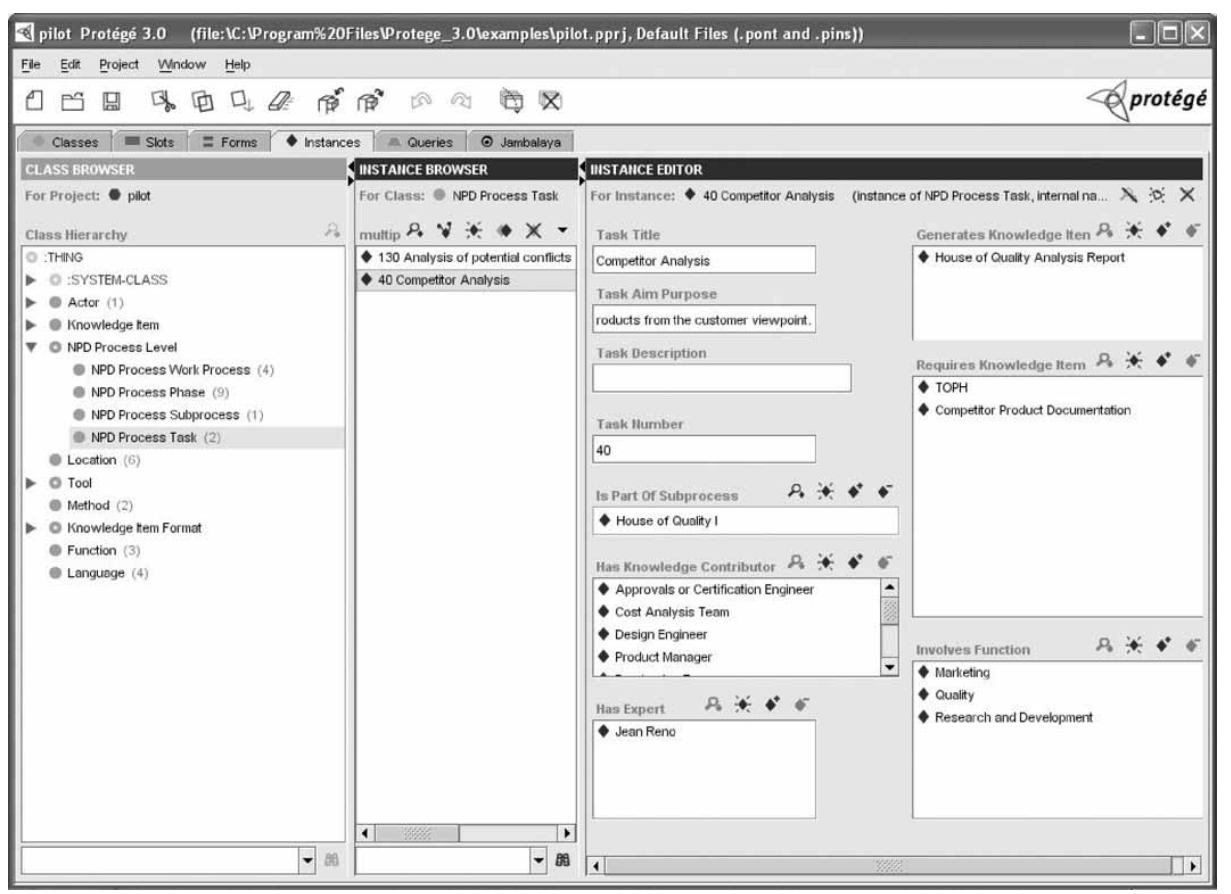

Figure 6. The main ontology browser window in the Protégé editor.

Dissemination of the knowledge is achieved using the web-based implementation of the ontology browser, a screenshot of which is shown in figure 8. As may be observed, the information contained in the forms is reproduced in the web tool. The ontology may be navigated using the ubiquitous 'point and click' paradigm.

\section{Conclusion}

Through application of the proposed methodology, ontology has been developed for the knowledge associated with a single task in the new product development process of the case study company. Additionally, simple mechanisms have been provided to visualize and disseminate this ontology. The resulting knowledgebased tool illustrates how the proposed methodology may be used to facilitate knowledge sharing in the new product development process. Two parts of the methodology were left outside the scope of this pilot study. These were the provision of a mechanism to prioritize the task level knowledge and a mechanism to present the ontology in different languages via the web. Further research is required on two issues. The first issue is to add the components missing from the knowledge-based tool by providing mechanisms to prioritize task level knowledge and present the ontology in different languages via the web. The second issue is to develop the competency of the ontology and validate its usefulness by adding 


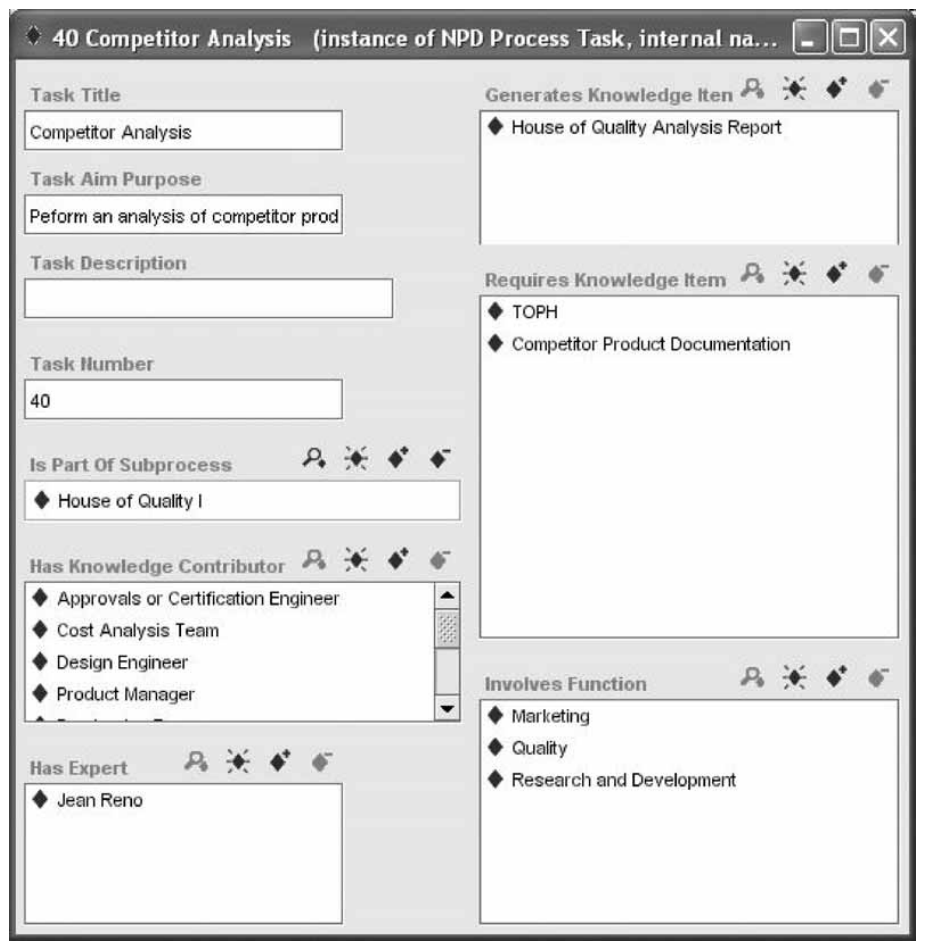

Figure 7. A form displaying information about the knowledge associated with a task.

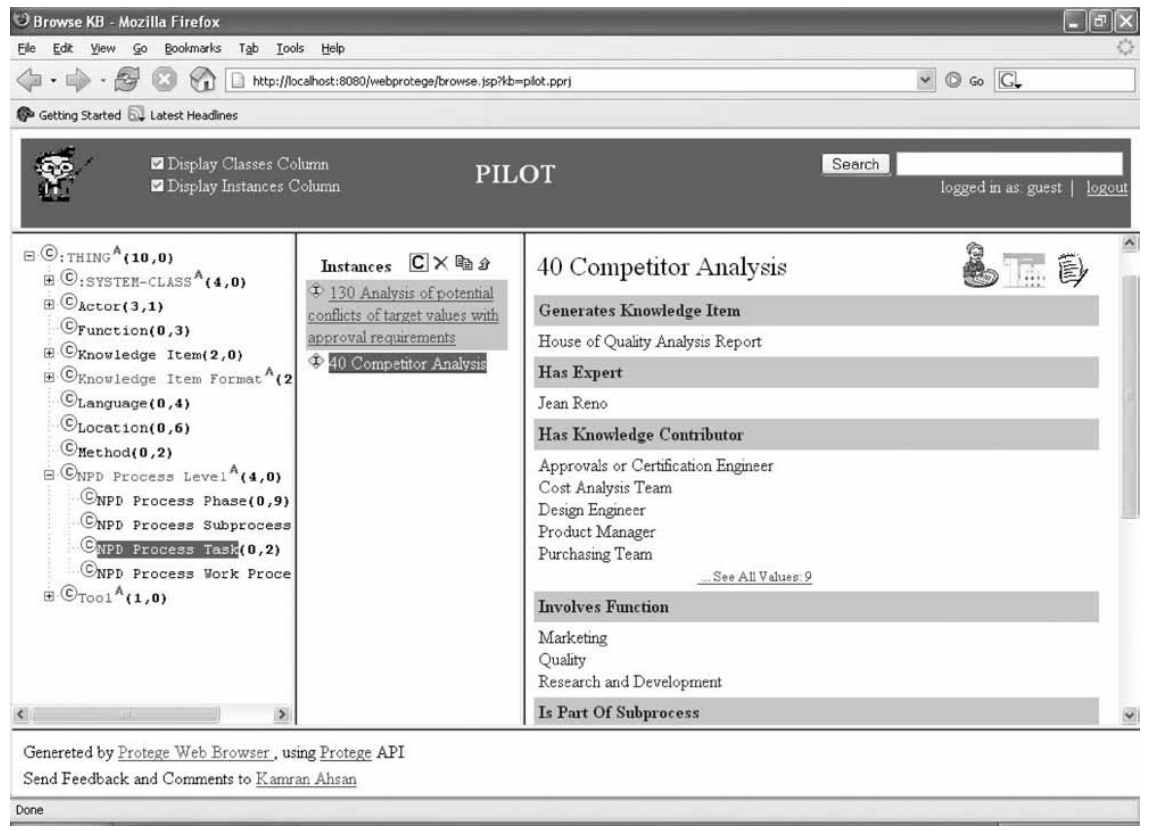

Figure 8. A web-based version of the knowledge-based tool using WebProtégé. 
information about more sub processes and tasks within the new product development process.

\section{Acknowledgements}

The authors would like to acknowledge the support of the EPSRC in conducting this research. They would also like to thank Ms Alexandra Brintrup and $\mathrm{Mr}$ Alvaro Camrillo for their contribution to the early stages of the company-based research project and knowledge audit study.

\section{References}

Akgün, A.E., Lynn, G.S. and Yilmaz, C., Learning process in new product development teams and effects on product success: a socio-cognitive perspective. Indust. Market. Manage., 2006, 35, 210-224.

Brown, S.L. and Eisenhardt, K.M., Product development: past research, present findings, and future directions. Acad. Manage. Rev., 1995, 20, 343-378.

Browning, T.R. and Eppinger, S.D., Modeling impacts of process architecture on cost and schedule risk in product development. IEEE Trans. Eng. Manage., 2002, 49, 428-442.

Carpuat, M., Ngai, G., Fung, P. and Church, K.W. Creating a bilingual ontology: a corpusbased approach for aligning WordNet and HowNet, in Proceedings of the 1st International WordNet Conference, 2002.

Corcho, O., Fernandez-Lopez, M. and Gomez-Perez, A., Methodologies, tools and languages for building ontologies. Where is their meeting point? Data \& Knowledge Eng., 2003, 46, $41-64$.

Debenham, J. and Clark, J., The knowledge audit. Robot. Comp.-Integ. Manuf., 1994, 11, 201-211.

Desouza, K. and Evaristo, R., Global knowledge management strategies. Euro. Manage. J., 2003, 21, 62-67.

Duineveld, A.J., Stoter, R., Weiden, M.R., Kenepa, B. and Benjamins, V.R., Wonder Tools? A comparative study of ontological engineering tools. Int. J. Human-Computer Studies, 2000, 52, 1111-1133.

Effendi, I., Henson, B., Agouridas, V. and de Pennington, A., Methods and tools for requirements engineering of made-to-order mechanical products, in ASME 2002 Design Engineering Technical Conferences and Computer and Information in Engineering Conference, 2002.

Eppinger, S.D., Innovation at the speed of information. Harv. Business Rev., 2001, 79, $149-158$.

Eppler, M.J., Seifried, P.M., and Röpnack, A., Improving knowledge intensive processes through an enterprise knowledge medium. Proceedings of the ACM SIGCPR Conference on Computer Personnel Research, 1999, pp. 222-230.

Ghoshal, S. and Bartlett, C.A., The multinational corporation as an interorganisational network. Acad. Manage. Rev., 1990, 15, 603-623.

Gieskes, J.F.B. and Langenberg, I., Learning and improvement in product innovation processes: enabling behaviours. Syst. Eng., 2001, 4, 134-144.

Gruber, T.R., A translation approach to portable ontology specifications. Knowl. Acquis., 1993, 5, 199-220.

Guyot, J., Radhouani, S. and Falquet, G., Ontology-based multilingual information retrieval, in Working Notes for the CLEF 2005 Workshop, 2005.

Holland, S., Gaston, K. and Gomes, J., Critical success factors for cross-functional teamwork in new product development. Int. J. Manage. Rev., 2000, 2, 231-259. 
Kerr, C.I., Roy, R. and Sackett, P.J., A product ontology for automotive seat specification, in ASME 2004 Design Engineering Technical Conferences and Computers and Information in Engineering Conference, 2004.

Kilker, J., Conflict on collaborative design teams. IEEE Tech. Soc. Mag., 1999, 18, 12-21.

Kleinsmann, M. and Valkenburg, R., Learning from collaborative new product development products. J. Workplace Learn., 2005, 17, 146-156.

Lambrix, P., Habbouche, M. and Perez, M., Evaluation of ontology development tools for bioinformatics. Bioinform., 2003, 19, 1564-1571.

Lauser, B., Wildemann, T., Poulos, A., Fisseha, F., Keiser, J. and Katz, S., A comprehensive framework for building multilingual domain ontologies: creating a prototype biosecurity ontology, in Proceedings of the International Conference on Dublin Core and Metadata for e-Communities, 2002, pp. 113-123.

Liebowitz, J., Rubenstein-Montano, B., McCaw, D., Buchwalter, J., Browning, C., Newman, B. and Rebeck, K., The knowledge audit. Knowl. Proc. Manage., 2000, 7, $3-10$.

Moore, J., Stader, J., Chung, P., Jarvis, P. and Macintosh, A., Ontologies to support the management of new product development in the chemical process industries, in International Conference on Engineering Design, 1999.

Morelli, M.D., Eppinger, S.D. and Gulati, R.K., Predicting technical communication in product development organisations. IEEE Transactions on Engineering Management, 1995, 42, 215-222.

Noy, N.F. and McGuinness, D.L., Ontology development 101: a guide to creating your first ontology. Available online at: http://www.ksl.stanford.edu/people/dlm/papers/ ontology101/ontology101-noy-mcguinness.html (accessed March 2005).

Noy, N.F., Fergerson, R.W. and Musen, M.A., The knowledge model of Protégé 2000: Combining interoperability and flexibility, in 2nd International Conference on Knowledge Engineering and Knowledge Management (EKAW'2000), 2000.

Pinto, H.S. and Martins, J.P., Ontologies: how can they be built? Knowledge and Information Systems, 2004, 6, 441-464.

Ramesh, B. and Tiwana, A., Supporting collaborative process knowledge management in new product development teams. Dec. Sup. Syst., 1999, 27, 213-235.

Rezayat, M., Knowledge-based product development using XML and KCs. CAD, 2000, 32, 299-309.

Stanford Medical Informatics, The Protégé ontology editor and knowledge acquisition system. Available online at: http://protege.stanford.edu/ (accessed February 2006a).

Stanford Medical Informatics, Protégé web browser. Available online at: http://protege. stanford.edu/plugins/protege_browser/index.html (accessed February 2006b).

Studer, R., Benjamins, V.R. and Fensel, D., Knowledge engineering: principles and methods. Data \& Knowledge Engineering, 1998, 25, 161-197.

Toye, G., Cutkosky, M.R., Leifer, L.J., Tenenbaum, J.M. and Glicksman, J., SHARE: a methodology and environment for collaborative product development. Int. J. Intel. Coop. Inform. Syst., 1994, 3, 129-153.

Ulrich, K.T. and Eppinger, S.D., Product Design and Development, 3rd ed., 2003 (McGraw-Hill: New York, NY).

Zahay, D., Griffin, A. and Fredericks, E., Sources, uses and forms of data in the new product development process. Indust. Market. Manage., 2004, 33, 657-666.

Zhang, S., Shen, W. and Ghenniwa, H., A review of internet-based product information sharing and visualisation. Comp. in Indust., 2004, 54, 1-15. 